See REVIEW page 252 See REVIEW page 261 See REVIEW page 271 See REVIEW page 279 See REVIEW page 288

\title{
The impact of mucosal infections on acquisition and progression of tuberculosis
}

\author{
S Perry ${ }^{1}$, R Hussain $^{2}$ and J Parsonnet ${ }^{1}$
}

More than one-third of the world's population, or over 2 billion people, are infected with Mycobacterium tuberculosis, the causative pathogen of tuberculosis in humans. Why only $10 \%$ of those infected develop active disease while the remainder harbor latent infection remains one of the greatest scientific and public health mysteries. Bacterial persistence is characterized by a dynamic state of immunological tolerance between pathogen and host. The critical role of CD4 ${ }^{+} \mathrm{T}$ cells in defense against intracellular pathogens became evident during epidemiological studies of HIV-1 infection, which showed a clear inverse relationship between CD4 ${ }^{+} \mathrm{T}$-cell count in peripheral blood and increased risk of infection with $M$. tuberculosis, pneumocystis and Toxoplasma gondii. There is also growing evidence of a common mucosal immune system, whereby immune cells activated at one mucosal site may disseminate to remote effector sites. In this commentary, we review emerging evidence from human studies that the outcome of $M$. tuberculosis infection is influenced by concurrent mucosal infections, using Helicobacter pylori and geohelminths as examples. Understanding how the complexity of microbial exposures influences host immunity may have important implications for vaccine development and therapeutic interventions.

\section{INTRODUCTION}

The mucosa of the gastrointestinal and respiratory tracts comprise the largest surfaces in contact with the external milieu. This system is designed to provide a physico-chemical barrier against dissemination of pathogenic mircroorganisms while sampling a vast array of foreign antigens, presenting them to the immune system, and adapting them to the presence of foreign microbial communities. ${ }^{1}$ In addition to pattern recognition and secretory antibody defenses, mucosalassociated lymphoid tissue (MALT) populated by distinct dendritic, T, B, and accessory cell populations-acts as an inductive tissue for priming of antigen-presenting cells at remote effector sites via the "common mucosal immune system". 2,3 Gut-associated lymyphoid tissue - the subset of MALT that exists in the gastrointestinal tract-is a major site for induction of T regulatory cells necessary for microbial tolerance. ${ }^{4}$ With recognition of the common mucosal immune system and its unique properties, the development of mucosal vaccines and therapies has become an area of intense research interest. ${ }^{2,5,6}$

In this commentary, we briefly review epidemiological evidence that the clinical outcome of the respiratory infection caused by Mycobacterium tuberculosis may be modulated by mucosal infections that are endemic in the same populations. As examples, we focus on Helicobacter pylori and gastro-intestinal helminthiasis, two major gut-associated "pathobiontic" infections that frequently co-exist in TBinfected hosts (Figure 1). We propose that colonization with these pathogens exerts competitive effects on regulation of the immune response to $M$. tuberculosis: H. pylori promoting a Th1-type response consistent with control of TB, and helminthiasis promoting a Th2-type response that may disregulate responses to tuberculosis (TB). In each case, the newly recognized Th17 lineage $\mathrm{e}^{7-10}$ may also have a role. These lines of investigation are just beginning, and further mechanistic as well as immuno-epidemiological research is needed. Understanding how common, naturally occurring mucosal infections influence immunity to TB may lead to further insights into the therapeutic properties of the common mucosal immune system.

${ }^{1}$ Division of Infectious Diseases and Geographic Medicine, Stanford University, Stanford, CA, USA. ²Department of Molecular Biology, Aga Khan University, Karachi, Pakistan. Correspondence: S Perry (shnperry@stanford.edu) 


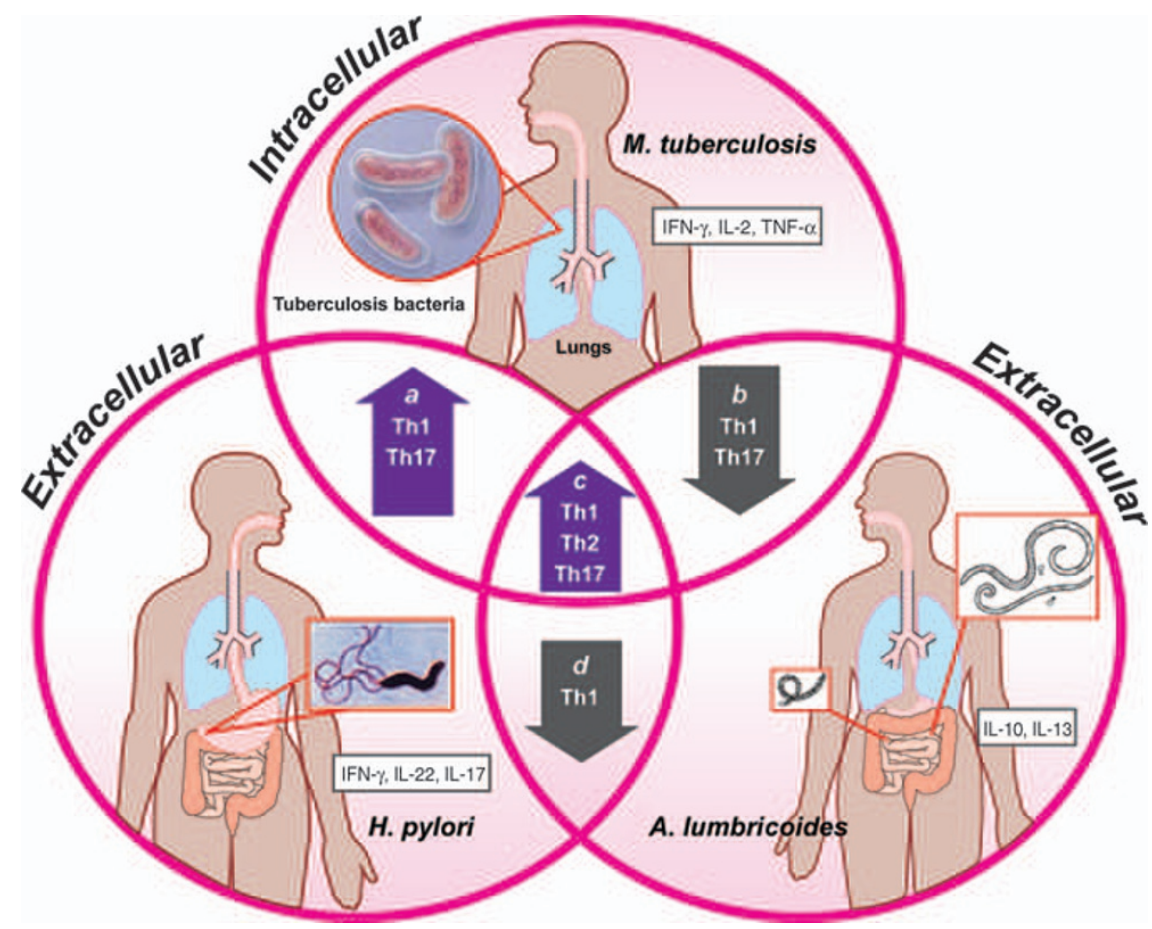

Figure 1 Hypothetical framework for interaction of protective immune responses to Mycobacterium tuberculosis (Mtb) infection in the setting of (a) H. pylori, (b) intestinal helminth, and (c) triple infection: (a) Mtb/H. pylori coinfection may enhance pro-inflammatory signals (e.g., interferon (IFN)- $\gamma$ ) involved in control of TB infection, ${ }^{68,85}$ while (b) Mtb/helminth co-infection may suppress pro-inflammatory responses to Mtb infection via the Th2 pathway. ${ }^{53,54}$ The presence of all three infections (c) would be expected to heighten pro-inflammatory and anti-inflammatory signals. Also, as shown in (d), H. pylori and helminth co-infection may suppress proinflammatory responses to H. pylori infection. ${ }^{81,82} \mathrm{IL}$, interleukin; TNF, tumor necrosis factor.

\section{IMMUNO-EPIDEMIOLOGY OFTB}

The intracellular pathogen $M$. tuberculosis has co-existed in human populations for at least 50,000 years. ${ }^{11}$ Spread from person to person via aerosol droplets, M. tuberculosis infects one-third of the world's population, or over 2 billion people. Despite dramatic declines in incidence and prevalence of TB infection in industrialized countries, there are over 8 million new cases of $M$. tuberculosis, 1.5 million deaths, and 40 million new infections each year. ${ }^{12}$ Although TB typically occurs in poor socio-economic conditions, even under these circumstances, a competent human immune system is equipped to control the infection. Of those exposed to TB, only $30 \%{ }^{13,14}$ are thought to develop the state of latent infection, during which the host remains clinically well, but bacilli survive within T-cell activated granulomas in a dormant or slowly replicating state. ${ }^{15}$ Of those harboring a latent infection, on average, only $5-10 \%$ will progress to active clinical disease, the majority within $2-3$ years of exposure. Approximately 50\% of those with active disease will survive without treatment, although each could infect an additional 10-20 contacts during the period of active disease. ${ }^{12,16}$

Protective immunity to TB is predominately cell-mediated and incompletely understood. Although the precise mechanisms are controversial, TLR-mediated innate immunity and microbicides present in the airways, and are likely to play a role in resistance during the initial phases of acute infection. ${ }^{17}$ The only vaccine currently in use, the live attenuated M. bovis-derived Bacille-CalmetteGuerin (BCG) vaccine, stimulates a wide spectrum of cellular immune responses, but has highly variable efficacy, and does not protect against adult pulmonary disease. ${ }^{18,19}$ Based on animal models of BCG vaccination, T-cell recall responses are delayed approximately 2 weeks postchallenge, representing a significant delay in T-cell priming. ${ }^{20}$ For this reason, mucosal Th17 vaccines based on early recruitment of neutrophils with production of interleukin (IL)-17 and mediators such as IL-23 in the alveolar passages are a promising area of investigation. ${ }^{9}$ Mucosally administered TB vaccines may offer properties superior to the intramuscular route. $^{21}$

With the establishment of latent M. tuberculosis infection, a network of pro-inflammatory and regulatory circuits is invoked to sequester bacilli-infected macrophages within granulomae. Both tumor necrosis factor- $\alpha$ and interferon (IFN)- $\gamma$ derived from activated $\mathrm{T}$ cells appear to be essential for maintaining the integrity of granulomae and preventing reactivation. ${ }^{22}$ Central memory T-cells expressing IL-2 also appear to be important for clearance of infection and resolution of fibrosis. ${ }^{23-25}$ To circumvent these defenses, M. tuberculosis inhibits phagolysomic fusion and acidification within macrophages. ${ }^{15,26}$ In household-contact biomarker studies, early progression has been associated with alterations in the production of regulatory Th-2 cytokines, such as IL-4 and IL-10. ${ }^{27,28}$ Thus, the balance of effector and suppressive immune responses appears to be important in the host's prolonged management of TB infection. ${ }^{29}$ In longitudinal studies, many acquired immuno-suppressive conditions are known to disrupt this balance 
and increase the risk of active disease, including HIV infection, ${ }^{30,31}$ malnutrition, ${ }^{32}$ Vitamin D deficiency, ${ }^{33}$ diabetes, ${ }^{34}$ and anti-tumor necrosis factor- $\alpha$ therapy. ${ }^{35}$ With insights from systems biology models, a dynamic spectrum of TB infection, continuously mediated by micro-environmental factors, has been postulated. ${ }^{17,36}$ Understanding the role of concurrent mucosal infections like $H$. pylori and gastro-intestinal helminthiasis may lead to better delineation of this spectrum.

\section{INTESTINAL HELMINTHS AND M.TUBERCULOSIS}

Over a quarter of the world's population is infected with the soil-transmitted intestinal helminths, including the roundworm Ascaris lumbricoides, the hookworms Necatoramericanus and Ancylostomaduodenale, the whipworm Trichuristrichiura, and Enterobiusvermicularis, and Strongyloides spp. ${ }^{37,38}$ Despite their species diversity, gastrointestinal helminths (and also many non-mucosalworms) elicit a stereotypic human immune response. Classically, this is characterized by secretion of IgE and IgG4 isotype with concurrent eosinophilia, ${ }^{39}$ and polarization of $\mathrm{CD} 4+$ T cells towards a Th2 phenotype, ${ }^{40-42}$ on which expulsion depends. ${ }^{43}$ This "modified" Th2 phenotype is associated with production of the cytokines IL-4, IL-9, IL-10, IL-13, ${ }^{44}$ as well as induction of Foxp $3+$ Treg cells and regulatory cytokines TGF- $\beta$ and IL-10. ${ }^{45}$ In endemic regions, worm burdens tend to peak by adolescence, suggesting the development of partial resistance with age. ${ }^{40}$ Conversely, the virtual disappearance of helminths from the human microbiome of high-income countries has been linked with the rise in atopic and auto-immune conditions, ${ }^{46}$ possibly due to effects on Treg induction. Based on these findings, helminth-based therapies for conditions such as multiple sclerosis and inflammatory bowel disease are an active area of investigation. ${ }^{47,48}$

Although there are few studies, epidemiological evidence suggests that helminth infections may diminish immune responses to TB antigens. In humans, chronic intestinal helminathiasis is associated with immune hypo-responsiveness, ${ }^{49,50}$ including a "bystander effect", to oral cholera ${ }^{51,52}$ and BCG vaccines. ${ }^{53}$ In a randomized control trial, Elias et al. ${ }^{54}$ reported that albendazole deworming was associated with significant improvements in purified protein derivative-induced IFN- $\gamma$ production following BCG vaccination of Ethiopian adults. In a second trial, this team also reported that co-infected adults not receiving anti-helminthic treatment had increased production of PPD-induced TGF- $\beta 3$ months following BCG vaccination. ${ }^{53}$ In a Brazilian cohort, TB patients with intestinal parasites had lower IFN- $\gamma$ and higher IL-10 levels compared with TB patients without intestinal parasites. ${ }^{55}$ In in vitro T-cell functional studies in blood from Indonesian school children, researchers found that geohelminthassociated regulatory $\mathrm{T}$-cell responses suppressed IFN- $\gamma$ responses to BCG and Plasmodium falciparum, an effect that was reversed with removal of $\mathrm{CD} 4+\mathrm{CD} 25^{\mathrm{hi}} \mathrm{T}$ cells. ${ }^{56}$ These studies offer evidence that both inflammatory and regulatory signals involved in control of TB infection can be modified by concurrent intestinal helminth infection. Some filarial worms and tissue-invasive cestodes, beyond the scope of this commentary, are also thought to affect responsiveness to mycobacterial antigens, although the mechanisms may differ. ${ }^{49}$ Although environmental mycobacteria are widely considered to block immune responses to $\mathrm{TB}$ antigens, ${ }^{57}$ their distribution does not account for the wide variability in responses to BCG. The role of concurrent helminth infections needs to be more systematically explored in the context of TB vaccine and immunogenicity trials.

\section{H. PYLORI AND M.TUBERCULOSIS}

H.pylori, a bacterium that colonizes the gastric mucosa and epithelial lining of the human stomach, remains one of the most common chronic mucosal infections in the world, ${ }^{58}$ infecting approximately $80 \%$ of those in TB-endemic regions. Typically acquired in early life via oral-oral or fecal-oral pathways, ${ }^{59}$ chronic infection is now known to be the preeminent cause of gastric cancer and peptic ulcer disease. ${ }^{6-62}$ All infected hosts develop a superficial gastritis that typically persists asymptomatically for many decades. In addition to specific IgA, IgM, and IgG responses, this gastritis is associated with induction of IL-8 by epithelial cells, ${ }^{63}$ as well as a locally vigorous, cell-mediated immune response, characterized by increased mucosal concentrations of the inflammatory cytokines IFN- $\gamma$, tumor necrosis factor- $\alpha$, IL- $1 \beta$, and IL- $6 .{ }^{64}$ The cytokine IL-12 is present in large numbers of mononuclear cells and appears to be involved in differentiating naive T-cells into a Th1 phenotype. ${ }^{65}$ Although the predominate profile during infection is inflammatory, the IL4 antagonist, IL-4 $\delta 2$, T-regulatory-activated IL-10, as well as B-cell responses, may be permissive for chronic $H$. pylori colonization via counter-regulatory mechanisms. ${ }^{66}$ The role of IL-17 in inflammation and recruitment of neutrophils at the site of infection is an area of intense investigation. ${ }^{67,68}$

As helminth infections vary with respect to their immunological effects, so too, $H$. pylori strains elicit different immune responses. ${ }^{69}$ Of particular note, some $H$. pylori strains contain a pathogenicity island, ${ }^{70}$ a 30 -gene cassette encoding a Type IV secretion system that translocates virulence factors to the host cytosol. ${ }^{71}$ This pathogenicity island induces increased inflammation with higher risk of ulcer disease ${ }^{72}$ and gastric cancers. ${ }^{73,74}$ Host polymorphisms are also thought to exacerbate this interaction. ${ }^{75}$ Other differences in $H$. pylori strain, such as expression of the active form of the immunogenic vacuolating antigen ${ }^{76}$ or preservation of the outer membrane protein BabA (which mediates adhesion to human $\mathrm{Le}^{\mathrm{b}}$ blood group antigens), may also influence immunological reactions by affecting attachment and neutrophil recruitment. ${ }^{64}$

Dramatic declines in the incidence of H. pylori infection occurring in industrialized regions during the twentieth century have corresponded with increases in the prevalence of allergy-like symptoms ${ }^{77}$ as well as upper gastrointestinal diseases. ${ }^{78}$ These trends have prompted some investigators to speculate that in regions of high infant mortality, immune-regulated $H$. pylori infection confers survival advantages against deadlier infections of early childhood. ${ }^{79}$ For example, in Northern 
Californian Hispanic households, $H$. pylori infection was associated with protection from household gastroenteritis. ${ }^{80}$ That concurrent helminth infection has been observed to alter the inflammatory response to $H$. pylori in rodent ${ }^{81}$ and human ${ }^{82}$ studies could support the notion that the two infections exert competitive regulatory effects on cellmediated immunity when present in the same host.

Because social conditions contributing to the prevalence of $M$. tuberculosis and H. pylori are similar, cross-sectional studies of disease association tend to yield conflicting results. ${ }^{83,84}$ Few studies have correlated immune responses to $H$. pylori and TB infection in co-infected hosts. In a preliminary finding, we have reported that Northern Californians seropositive for $H$. pylori and testing positive for latent TB have enhanced IFN- $\gamma$ production and a dominant Th1-type cytokine profile in response to specific TB antigens. ${ }^{85} \mathrm{We}$ also reported that, compared with latently infected household contacts who did not progress to active $\mathrm{TB}$ disease during 2 years of follow-up, Gambian TB patients were one-third as likely to be $H$. pylori seropositive. However, in Gambian and Pakistani household contacts exposed to an infectious case of TB, baseline $H$. pylori infection was not associated with secondary case activation after 2 years. In cynomolgus macaques, those with natural $H$. pylori infection were one-third as likely to progress to TB 6-8 months after M. tuberculosis challenge. ${ }^{85}$ Although these lines of investigation provide preliminary evidence of enhanced immunity to TB with $H$. pylori infection, challenges in study design remain to be overcome. These include the relatively poor predictive value of immunodiagnostics in the setting of primary progressive $\mathrm{TB},{ }^{86}$ the need to identify more prevalent intermediate markers of progression, and the suboptimal performance of $H$. pylori serology in the developing world. ${ }^{85}$

If $H$. pylori influences the immune response to $\mathrm{TB}$ infection, there are several possible mechanisms. For example, H. pylori could promote a "Th2-Th1 switch" in early life, such that Th1-type responses to an unrelated MALT infection are permanently heightened. Such a "hygiene hypothesis" has been proposed for hepatitus A virus. ${ }^{87}$ Studies comparing BCG immunogenicity in infants and adolescents from developing and industrialized countries also support this type of phenomenon. ${ }^{88}$ In a second model, active, asymptomatic, $H$. pylori gastritis would provide a continuous source of inflammatory stimulation. Such a "bystander" model was recently described for mice infected with mucosal herpes viridae and protected from subsequent challenge by $Y$. pestis and L. monocytogenes. This model may apply uniquely to chronic mucosal infections. ${ }^{89}$ Though more speculative, H. pylori-induced IL-17 might have a role in early T-cell recruitment to the lung compartment, thereby enhancing initial resistance to latent TB infection. Another possibility is that dysregulation of the $H$. pylori-associated gastric T-regulatory system compromises systemic Th 1 responses involved in control of a TB infection. In separate laboratory studies, the IL-4 inhibitor, IL4 $\delta 2$ splice variant, has been implicated both in upregulation of the Th1 dominant $H$. pylori Cag A response ${ }^{90}$ and downregulation of the Th2-mediated response associated with progression of latent $\mathrm{TB}$ infection. ${ }^{91}$

\section{TOWARD A MICROBIOMIC PERSPECTIVE}

The human immune system has evolved in the face of a panoply of commensal and mutual infections. ${ }^{92}$ Classic mucosal defenses, including organogenesis of lymphoid follicles, induction of secretory $\operatorname{IgA}$, and recruitment of the cell-mediated armentarium appear to be seeded and orchestrated by the establishment of microbrial communities in early life. ${ }^{93,94}$ Growing evidence implicates the human microbiome as a major regulator of immunopathologies associated with diverse conditions such as inflammatory bowel disease, ${ }^{95}$ obesity,${ }^{96}$ allergies,${ }^{97}$ and even psychopathology. ${ }^{98}$ Understanding the interactions of naturally occurring mucosal infections such as $H$. pylori, intestinal helminths, and M. tuberculosisthree distinct parasitic infections present in the same ecosystem for millennia, exerting contrasting immunoregulatory effects, to which the normal human immune is substantially adapted-offers a potentially informative "microbiomic" perspective for vaccine and immunodiagnostic research. The evidence presented here remains preliminary and belies the need for more original work. The research infrastructure now assembled for $\mathrm{TB}$ vaccine trials provides a well-organized platform to roll out interdisciplinary prospective studies of major public health importance.

\section{ACKNOWLEDGMENTS}

This work was supported by the National Institutes of Health (NIH) K23 Al054443 (SP), R01-42081 (JP), and the Bill and Melinda Gates Foundation.

\section{DISCLOSURE}

The authors declared no conflict of interest.

C) 2011 Society for Mucosal Immunology

\section{REFERENCES}

1. Cerf-Bensussan, N. \& Gaboriau-Routhiau, V. The immune system and the gut microbiota: friends or foes? Nat. Rev. 10, 735-744 (2010).

2. Holmgren, J. \& Czerkinsky, C. Mucosal immunity and vaccines. Nat. Med. 11, S45-S53 (2005).

3. Mestecky, J. The common mucosal immune system and current strategies for induction of immune responses in external secretions. J. Clin. Immunol. 7, 265-276 (1987).

4. Grainger, J.R., Hall, J.A., Bouladoux, N., Oldenhove, G. \& Belkaid, Y. Microbe-dendritic cell dialog controls regulatory $\mathrm{T}$-cell fate. Immunol. Rev. 234, 305-316 (2010).

5. Chen, K. \& Cerutti, A. Vaccination strategies to promote mucosal antibody responses. Immunity 33, 479-491 (2010).

6. Neutra, M.R. \& Kozlowski, P.A. Mucosal vaccines: the promise and the challenge. Nat. Rev. 6, 148-158 (2006).

7. Blaschitz, C. \& Raffatellu, M. Th17 cytokines and the gut mucosal barrier. J. Clin. Immunol. 30, 196-203 (2010).

8. Dubin, P.J. \& Kolls, J.K. Th17 cytokines and mucosal immunity. Immunol. Rev. 226, 160-171 (2008).

9. Khader, S.A., Gaffen, S.L. \& Kolls, J.K. Th17 cells at the crossroads of innate and adaptive immunity against infectious diseases at the mucosa. Mucosal Immunol. 2, 403-411 (2009).

10. Liu, J.Z., Pezeshki, M. \& Raffatellu, M. Th17 cytokines and host-pathogen interactions at the mucosa: dichotomies of help and harm. Cytokine 48, 156-160 (2009).

11. Hershberg, R. et al. High functional diversity in Mycobacterium tuberculosis driven by genetic drift and human demography. PLOS Biol. 6 , e311 (2008).

12. World Health Organization. Global TB Control: Surveillance, Planning, Financing (World Health Organization, Geneva 2008).

13. Shaw, J.B. \& Wynn-Williams, N. Infectivity of pulmonary tuberculosis in relation to sputum status. Am. Rev. Tuberc. 69, 724-732 (1954).

14. Jereb, J., Etkind, S.C., Joglar, O.T., Moore, M. \& Taylor, Z. Tuberculosis contact investigations: outcomes in selected areas of the United States, 1999. Int. J. Tuberc. Lung Dis. 7, S384-S390 (2003). 
15. Saunders, B.M. \& Britton, W.J. Life and death in the granuloma: immunopathology of tuberculosis. Immunol. Cell Biol. 85, 103-111 (2007).

16. Comstock, G.W. \& Cauthen, G.M. Epidemiology of tuberculosis. In Tuberculosis: A Comprehensive Approach (Reichman, L. \& Herschfield, E., eds) 23-48 (Marcel Decker, New York, NY, 1993).

17. Lin, P.L. \& Flynn, J.L. Understanding latent tuberculosis: a moving target. J. Immunol. 185 , 15-22 (2010)

18. Reece, S.T. \& Kaufmann, S.H. Rational design of vaccines against tuberculosis directed by basic immunology. Int. J. Med. Microbiol. 298, 143-150 (2008).

19. Colditz, G.A. et al. The efficacy of bacillus Calmette-Guerin vaccination of newborns and infants in the prevention of tuberculosis: metaanalyses of the published literature. Pediatrics 96, 29-35 (1995).

20. Cooper, A.M., Callahan, J.E., Keen, M., Belisle, J.T. \& Orme, I.M. Expression of memory immunity in the lung following re-exposure to Mycobacterium tuberculosis. Tuber Lung Dis. 78, 67-73 (1997).

21. Dietrich, J. et al. Mucosal administration of Ag85B-ESAT-6 protects against infection with Mycobacterium tuberculosis and boosts prior bacillus Calmette-Guerin immunity. J. Immunol. 177, 6353-6360 (2006).

22. Flynn, J.L. \& Chan, J. Immunology of tuberculosis. Annu. Rev. Immunol. 19, 93-129 (2001).

23. Millington, K.A. et al. Dynamic relationship between IFN-gamma and IL-2 profile of Mycobacterium tuberculosis-specific T cells and antigen load. J. Immunol. 178, 5217-5226 (2007).

24. Millington, K.A., Gooding, S., Hinks, T.S., Reynolds, D.J. \& Lalvani, A. Mycobacterium tuberculosis-specific cellular immune profiles suggest bacillary persistence decades after spontaneous cure in untreated tuberculosis. J. Infect. Dis. 202, 1685-1689 (2010).

25. Biselli, R. et al. Detection of interleukin-2 in addition to interferon-gamma discriminates active tuberculosis patients, latently infected individuals, and controls. Clin. Microbiol. Infect. 16, 1282-1284 (2010)

26. Russell, D.G. Mycobacterium tuberculosis: here today, and here tomorrow. Nat. Rev. Mol. Cell Biol. 2, 569-577 (2001).

27. Hussain, R., Talat, N., Shahid, F. \& Dawood, G. Longitudinal tracking of cytokines after acute exposure to tuberculosis: association of distinct cytokine patterns with protection and disease development. Clin. Vaccine Immunol. 14, 1578-1586 (2007).

28. Hussain, R. et al. Cytokine profiles using whole-blood assays can discriminate between tuberculosis patients and healthy endemic controls in a BCG-vaccinated population. J. Immunol. Methods 264, 95-108 (2002).

29. Ehlers, S. Lazy, dynamic or minimally recrudescent? On the elusive nature and location of the mycobacterium responsible for latent tuberculosis. Infection 37, 87-95 (2009).

30. Corbett, E.L. et al. The growing burden of tuberculosis: global trends and interactions with the HIV epidemic. Arch. Intern. Med. 163, 1009-1021 (2003).

31. Elliott, A.M. et al. Cytokine responses and progression to active tuberculosis in HIV-1infected Ugandans: a prospective study. Trans.
R. Soc. Trop. Med. Hygiene 98, 660-670 (2004).

32. Moran-Mendoza, O., Marion, S.A., Elwood, K., Patrick, D. \& FitzGerald, J.M. Risk factors for developing tuberculosis: a 12-year follow-up of contacts of tuberculosis cases. Int. J. Tuberc. Lung Dis. 14, 1112-1119 (2010).

33. Talat, N., Perry, S., Parsonnet, J., Dawood, G. \& Hussain, R. Vitamin d deficiency and tuberculosis progression. Emerg. Infect. Dis. 16, 853-855 (2010).

34. Blumberg, H.M. et al. American Thoracic Society/Centers for Disease Control and Prevention/Infectious Diseases Society of America: treatment of tuberculosis. Am. J. Respir. Crit. Care Med. 167, 603-662 (2003).

35. Jacobs, M. et al. Tumor necrosis factor is critical to control tuberculosis infection. Microbes Infect./Inst. Pasteur 9, 623-628 (2007).

36. Barry, C.E. III et al. The spectrum of latent tuberculosis: rethinking the biology and intervention strategies. Nat. Rev. Microbiol. 7, 845-855 (2009).

37. Hotez, P.J. et al. Helminth infections: the great neglected tropical diseases. J. Clin. Invest. 118, 1311-1321 (2008)

38. Cooper, P.J. Mucosal immunology of geohelminth infections in humans. Mucosal Immunol. 2, 288-299 (2009).

39. Klion, A.D. \& Nutman, T.B. The role of eosinophils in host defense against helminth parasites. J. Allergy Clin. Immunol. 113, 30-37 (2004).

40. Bourke, C.D., Maizels, R.M. \& Mutapi, F. Acquired immune heterogeneity and its sources in human helminth infection. Parasitology 138, 1-21 (2010).

41. Maizels, R.M. \& Yazdanbakhsh, M. Immune regulation by helminth parasites: cellular and molecular mechanisms. Nat. Rev. 3, 733-744 (2003).

42. Cooper, P.J. et al. Human infection with Ascaris lumbricoides is associated with a polarized cytokine response. J. Infect. Dis. 182, 12071213 (2000)

43. Jackson, J.A., Friberg, I.M., Little, S. \& Bradley, J.E. Review series on helminths, immune modulation and the hygiene hypothesis: immunity against helminths and immunological phenomena in modern human populations: coevolutionary legacies? Immunology 126, 18-27 (2009).

44. Turner, J.D. et al. Th2 cytokines are associated with reduced worm burdens in a human intestinal helminth infection. J. Infect. Dis. $\mathbf{1 8 8 ,}$ 1768-1775 (2003).

45. Grainger, J.R. et al. Helminth secretions induce de novo $T$ cell Foxp3 expression and regulatory function through the TGF-beta pathway. J. Exp. Med. 207, 2331-2341 (2010).

46. Maizels, R.M. Infections and allergyhelminths, hygiene and host immune regulation. Curr. Opin. Immunol. 17, 656-661 (2005).

47. Osada, Y. \& Kanazawa, T. Parasitic helminths: new weapons against immunological disorders. J. Biomed. Biotechnol. 2010, 743758 (2010).

48. Erb, K.J. Can helminths or helminth-derived products be used in humans to prevent or treat allergic diseases? Trends Immunol. 30, 75-82 (2009).

49. van Riet, E., Hartgers, F.C. \& Yazdanbakhsh, M. Chronic helminth infections induce immunomodulation: consequences and mechanisms. Immunobiology 212, 475-490 (2007).

50. Borkow, G. et al. Chronic immune activation associated with intestinal helminth infections results in impaired signal transduction and anergy. J. Clin Invest. 106, 1053-1060 (2000).

51. Cooper, P.J. et al. Human infection with Ascaris lumbricoides is associated with suppression of the interleukin-2 response to recombinant cholera toxin B subunit following vaccination with the live oral cholera vaccine CVD 103HgR. Infect. Immunity 69, 1574-1580 (2001).

52. Cooper, P.J. et al. Albendazole treatment of children with ascariasis enhances the vibriocidal antibody response to the live attenuated oral cholera vaccine CVD 103-HgR J. Infect. Dis. 182, 1199-1206 (2000).

53. Elias, D., Britton, S., Aseffa, A., Engers, H. \& Akuffo, H. Poor immunogenicity of BCG in helminth infected population is associated with increased in vitro TGF-beta production. Vaccine 26, 3897-3902 (2008).

54. Elias, D. et al. Effect of deworming on human $T$ cell responses to mycobacterial antigens in helminth-exposed individuals before and after bacille Calmette-Guerin (BCG) vaccination. Clin. Exp. Immunol. 123, 219-225 (2001).

55. Resende Co, T., Hirsch, C.S., Toossi, Z., Dietze, R. \& Ribeiro-Rodrigues, R. Intestinal helminth co-infection has a negative impact on both anti-Mycobacterium tuberculosis immunity and clinical response to tuberculosis therapy. Clin. Exp. Immunol. 147, 45-52 (2007).

56. Wammes, L.J. et al. Regulatory T cells in human geohelminth infection suppress immune responses to BCG and Plasmodium falciparum. Eur. J. Immunol. 40, 437-442 (2010).

57. Black, G.F. et al. Patterns and implications of naturally acquired immune responses to environmental and tuberculous mycobacterial antigens in northern Malawi. J. Infect. Dis. 184, 322-329 (2001).

58. Perry, S., de Martel, C. \& Parsonnet, J. Helicobacter pylori. In Bacterial Infections of Humans (Brachman, P.S. \& Brutyn, E.A., eds) (Springer: Philadelphia, PA, 2009).

59. Brown, L.M. Helicobacter pylori: epidemiology and routes of transmission. Epidemiol. Rev. 22, 283-297 (2000).

60. Parsonnet, J. et al. Helicobacter pylori infection and the risk of gastric carcinoma. N. Engl. J. Med. 325, 1127-1131 (1991).

61. Parsonnet, J. et al. Helicobacter pylori infection and gastric lymphoma. N. Engl. J. Med. 330, 1267-1271 (1994).

62. Uemura, N. et al. Helicobacter pylori infection and the development of gastric cancer. N. Engl. J. Med. 345, 784-789 (2001).

63. Crabtree, J.E. et al. Helicobacter pylori induced interleukin-8 expression in gastric epithelial cells is associated with CagA positive phenotype. J. Clin. Pathol. 48, 41-45 (1995).

64. van Amsterdam, K., van Vliet, A.H., Kusters, J.G. \& van der Ende, A. Of microbe and man: determinants of Helicobacter pylori-related diseases. FEMS Microbiol. Rev. 30, 131-156 (2006).

65. D'Elios, M.M., Amedei, A., Benagiano, M., Azzurri, A. \& Del Prete, G. Helicobacter pylori, T cells and cytokines: the "dangerous liaisons". FEMS Immunol. Med. Microbiol. 44, 113-119 (2005). 
66. Holck, S. et al. Gastric mucosal cytokine responses in Helicobacter pylori-infected patients with gastritis and peptic ulcers. Association with inflammatory parameters and bacteria load. FEMS Immunol. Med. Microbiol. 36, 175-180 (2003).

67. Aujla, S.J., Dubin, P.J. \& Kolls, J.K. Th17 cells and mucosal host defense. Semin. Immunol. 19, 377-382 (2007).

68. Luzza, F. et al. Up-regulation of IL-17 is associated with bioactive IL-8 expression in Helicobacter pylori-infected human gastric mucosa. J. Immunol. 165, 5332-5337 (2000).

69. Blaser, M.J. Heterogeneity of Helicobacter pylori. Eur. J. Gastroenterol. Hepatol. 9 (Suppl 1), S3-S6 discussion S6-7 (1997).

70. Christie, P.J. \& Vogel, J.P. Bacterial type IV secretion: conjugation systems adapted to deliver effector molecules to host cells. Trends Microbiol. 8, 354-360 (2000).

71. Viala, J. et al. Nod1 responds to peptidoglycan delivered by the Helicobacter pylori cag pathogenicity island. Nat. Immunol. 5, 1166-1174 (2004).

72. Nomura, A.M., Perez-Perez, G.I., Lee, J., Stemmermann, G. \& Blaser, M.J. Relation between Helicobacter pylori cagA status and risk of peptic ulcer disease. Am. J. Epidemiol. 155, 1054-1059 (2002).

73. Parsonnet, J., Friedman, G.D., Orentreich, N. \& Vogelman, H. Risk for gastric cancer in people with CagA positive or CagA negative Helicobacter pylori infection. Gut 40, 297-301 (1997).

74. Peek, R.M. Jr. et al. Role of Helicobacter pylori $\operatorname{cagA}(+)$ strains and specific host immune responses on the development of premalignant and malignant lesions in the gastric cardia. Int. J. Cancer 82, 520-524 (1999).

75. El-Omar, E.M. et al. The role of interleukin-1 polymorphisms in the pathogenesis of gastric cancer. Nature 412, 99 (2001).

76. Atherton, J.C. et al. Mosaicism in vacuolating cytotoxin alleles of Helicobacter pylori. Association of specific vacA types with cytotoxin production and peptic ulceration. J. Biol. Chem. 270, 17771-17777 (1995).

77. Blaser, M.J., Chen, Y. \& Reibman, J. Does Helicobacter pylori protect against asthma and allergy? Gut 57, 561-567 (2008).

78. de Martel, C. et al. Helicobacter pylori infection and the risk of development of esophageal adenocarcinoma. J. Infect. Dis. 191, 761-767 (2005).

79. Blaser, M.J. \& Atherton, J.C. Helicobacter pylori persistence: biology and disease. J. Clin Invest. 113, 321-333 (2004).

80. Perry, S. et al. Helicobacter pylori and risk of gastroenteritis. J. Infect. Dis. 190, 303-310 (2004).

81. Fox, J.G. et al. Concurrent enteric helminth infection modulates inflammation and gastric mmune responses and reduces helicobacterinduced gastric atrophy. Nat. Med. 6, 536-542 (2000).

82. Whary, M.T. et al. Intestinal helminthiasis in Colombian children promotes a Th2 response to Helicobacter pylori: possible implications for gastric carcinogenesis. Cancer Epidemiol. Biomarkers Prev. 14, 1464-1469 (2005).

83. Sanaka, M. et al. No difference in seroprevalences of Helicobacter pylori infection between patients with pulmonary tuberculosis and those without. J. Clin. Gastroenterol. 27, 331-334 (1998)

84. Torres, M.A. et al. No association between Helicobacter pylori and Mycobacterium tuberculosis infections among gastrointestinal clinic attendees in Lima, Peru. Epidemiol. Infect. 130, 87-91 (2003).

85. Perry, S. et al. Infection with Helicobacter pylori is associated with protection against tuberculosis. PLoS One 5, e8804 (2010).

86. Hill, P.C. et al. Incidence of tuberculosis and the predictive value of ELISPOT and Mantoux tests in Gambian case contacts. PLoS One 3, e1379 (2008).

87. Umetsu, D.T., McIntire, J.J. \& DeKruyff, R.H $\mathrm{TIM}-1$, hepatitis $\mathrm{A}$ virus and the hygiene theory of atopy: association of TIM-1 with atopy. J. Pediatr. Gastroenterol. Nutr. 40 (Suppl 1), S43 (2005).

88. Black, G.F. et al. BCG-induced increase in interferon-gamma response to mycobacterial antigens and efficacy of BCG vaccination in Malawi and the UK: two randomised controlled studies. Lancet 359, 1393-1401 (2002).

89. Barton, E.S. et al. Herpesvirus latency confers symbiotic protection from bacterial infection. Nature 447, 326-329 (2007).

90. Orsini, B. et al. Helicobacter pylori cag pathogenicity island is associated with reduced expression of interleukin-4 (IL-4) mRNA and modulation of the IL-4delta2 mRNA isoform in human gastric mucosa. Infect. Immunity $\mathbf{7 1}$, 6664-6667 (2003).

91. Demissie, A. et al. Healthy individuals that control a latent infection with Mycobacterium tuberculosis express high levels of Th1 cytokines and the IL-4 antagonist IL-4delta2. J. Immunol. 172, 6938-6943 (2004).

92. Dethlefsen, L., McFall-Ngai, M. \& Relman, D.A. An ecological and evolutionary perspective on human-microbe mutualism and disease. Nature 449, 811-818 (2007).

93. Noverr, M.C. \& Huffnagle, G.B. Does the microbiota regulate immune responses outside the gut? Trends Microbiol. 12, 562-568 (2004).

94. Round, J.L. \& Mazmanian, S.K. The gut microbiota shapes intestinal immune responses during health and disease. Nat. Rev. 9, 313-323 (2009).

95. Bouma, G. \& Strober, W. The immunological and genetic basis of inflammatory bowel disease. Nat. Rev. 3, 521-533 (2003).

96. Turnbaugh, P.J. et al. An obesity-associated gut microbiome with increased capacity for energy harvest. Nature 444, 1027-1031 (2006).

97. Wills-Karp, M., Santeliz, J. \& Karp, C.L. The germless theory of allergic disease: revisiting the hygiene hypothesis. Nat. Rev. 1, 69-75 (2001).

98. Rook, G.A. \& Lowry, C.A. The hygiene hypothesis and psychiatric disorders. Trends Immunol. 29, 150-158 (2008). 